\title{
STM Light Emission Spectroscopy of Individual Quantum Wells: Measurement of Transport Parameters in Real Space
}

\author{
S. Ushioda*, T. Tsuruoka*, Y. Ohizumi ${ }^{\dagger}$, and H. Hashimoto ${ }^{\dagger}$ \\ *Photodynamics Research Center, RIKEN, Sendai 980-0845, Japan \\ ${ }^{\dagger}$ Research Institute of Electrical Communication, Tohoku University, Sendai 980-8577, Japan
}

Received on 31 March, 2003

\begin{abstract}
By spectroscopically analyzing the light emitted from the tip-sample gap of the scanning tunneling microscope (STM), we have investigated the carrier transport as well as the luminescence properties of AlGaAs/GaAs quantum wells (QW's). The emission intensity form a target well was measured as a function of the tip position on a cleaved (110) surface of the QW structures. The thermalization length and the diffusion length of the injected electrons were determined in real space.
\end{abstract}

\section{Introduction}

Visible light is emitted when electrons or holes are injected from the tip of the scanning tunneling microscope (STM). By spectroscopic analysis of this emission, one can characterize nanometer scale objects (nanostructures) with atomicscale spatial resolution. We are utilizing this effect to study the electronic transitions in individual surface nanostructures such as quantum wells (QW's), quantum dots (QD's), and atoms and molecules adsorbed on solid surfaces. In this method one first observes the STM image of the surface topography and finds the nanostructures of interest. Then the STM tip is located over the target structure and the tunneling current is injected into the specific individual target. The spectrum of the emitted light is measured with the STM tip fixed over the target, thus obtaining information on the electronic transitions of the individual target nanostructure. This method is shown schematically in Fig. 1.

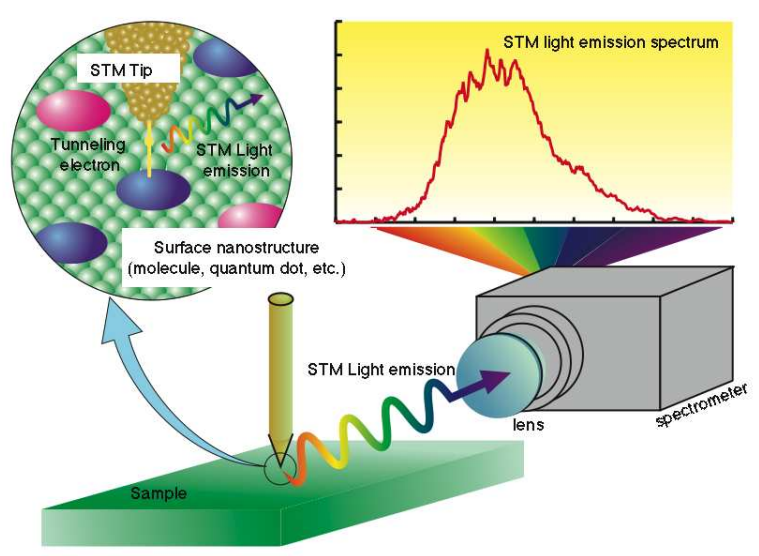

Figure 1. Experimental concept of STM light emission.

We have applied this spectroscopic technique with an atomic scale spatial resolution to several sample systems, including QW's [1,2], QD's [3], and surface adsorbed atoms and molecules [4,5]. In this talk we will focus on the measurement of the electron thermalization and diffusion lengths in real space in $\mathrm{AlGaAs} / \mathrm{GaAs} \mathrm{QW}$ structures [6,7]. The details of the sample and STM setup are shown in Fig. 2.

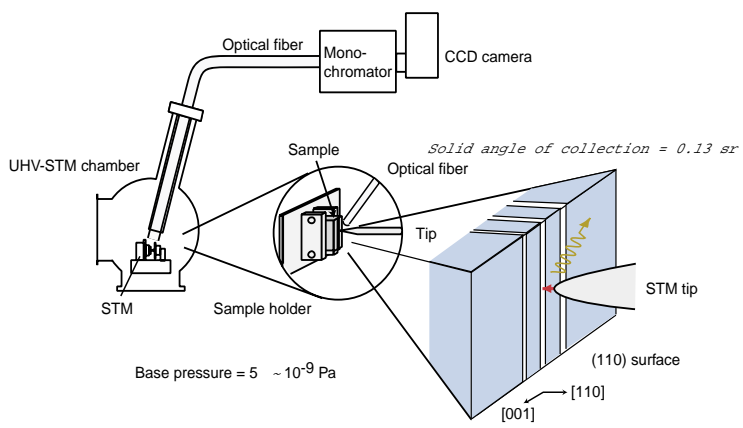

Figure 2. Setup for STM light emission spectroscopy.

\section{Experiment and Results}

The sample was grown by molecular-beam epitaxy (MBE) on a p-type $\mathrm{GaAs}(001)$ substrate. It had p-GaAs QW's of widths 3.1, 5.1, and $10.2 \mathrm{~nm}$ sandwiched between the barrier layers of $\mathrm{p}-\mathrm{Al}_{0.3} \mathrm{Ga}_{0.7} \mathrm{As}$ whose width was $50 \mathrm{~nm}$. The hole concentration was $2 \times 10^{19} \mathrm{~cm}^{-3}$ for the GaAs QW, and $1.4 \times 10^{19} \mathrm{~cm}^{-3}$ for the AlGaAs barrier layers. This sample was cleaved under ultra-high vacuum (UHV) to expose the clean (110) surface. The STM image of this surface showed the QW layers as light stripes as seen in Fig. 3. The STM tip injected electrons into this surface at different distances from the QW's. The light emission spectra and their intensity were measured as a function of the location of the tip. 


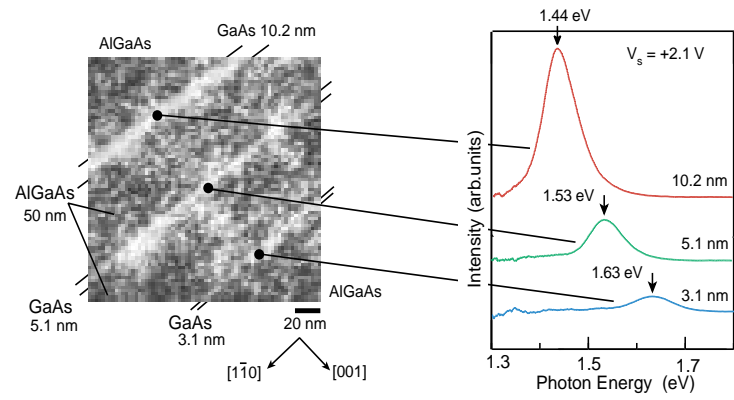

Figure 3. STM image of cleaved AlGaAs/GaAs QW structures and light emission spectra measured over the three wells.

The emission spectra from the three wells are shown in Fig. 3. We see that the peak energy shifts to higher energies as the well width decreases. This is a direct demonstration of the quantum confinement effect. Since the peak energies of the emission from the different wells can be clearly identified, one can know the relative number of electrons that reach each well from the intensity of the corresponding peak. We measured the intensity from different wells as a function of the distance between the injection point and the position of the wells. The emission intensity vs. distance is plotted for the $10.2 \mathrm{~nm}$ QW in Fig. 4. The intensity decay curve can be fitted by assuming two exponential decay lengths $L_{1}$ and $L_{2}$ as shown in Fig. 4.

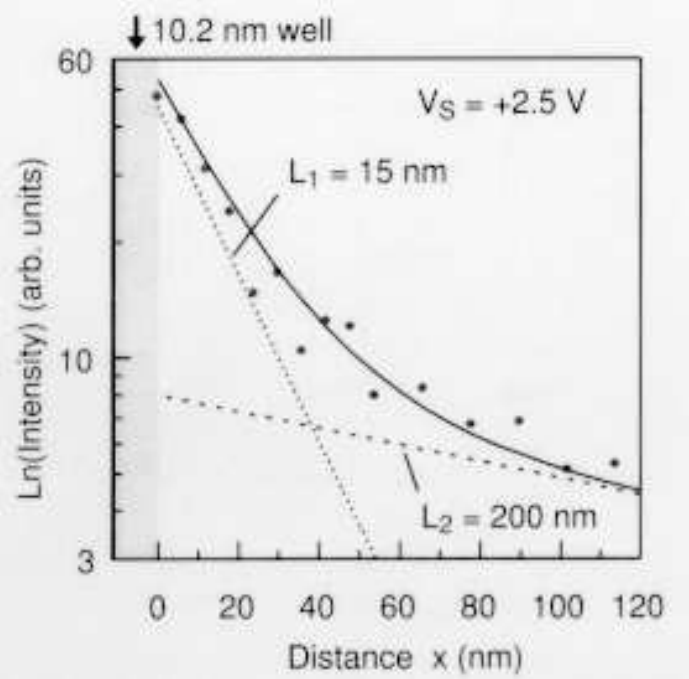

Figure 4. Logarithmic plot of emission intensity from $10.2 \mathrm{~nm}$ well as a function of tip position. The dotted and dashed lines are the exponential decay functions with different decay constants $\mathrm{L}_{1}$ and $\mathrm{L}_{2}$. The solid curve is the superposition of the dotted and dashed lines.

We identified these two decay distances as the thermalization length and the diffusion length by comparing the experimental decay curve with the results of Monte Carlo simulation. The thermalization length is the mean distance that the injected electron travels before thermally relaxing to the bottom of the conduction band through scattering processes with phonons, optical phonon-plasmon coupled modes, ionized impurities, etc. The diffusion length is the mean distance that the electron propagates before getting lost by recombination processes. Fig. 5 shows the electron number decay curves for the three wells when the bias voltage $\mathrm{V}_{S}$ is +2.5 V. Fig. 6 shows the corresponding data for $\mathrm{V}_{S}=+2.1$ V.

By comparing the data in Figs. 5 and 6, we see that the thermalization length depends on the electron injection energy that is determined by the bias voltage of the tunneling, i. e. on the energy level at which electrons tunnel into the conduction band. When the injection energy gets higher $\left(\mathrm{V}_{S}\right.$ $=+2.5 \mathrm{~V}$ ), the thermalization length gets longer than for the case of lower injection energy $\left(\mathrm{V}_{S}=+2.1 \mathrm{~V}\right)$. On the other hand the diffusion length is independent of the bias voltage. This result is reasonable, when one realizes that higher energy electrons will take a larger number of scattering events before they thermalize down to the bottom of the conduction band.

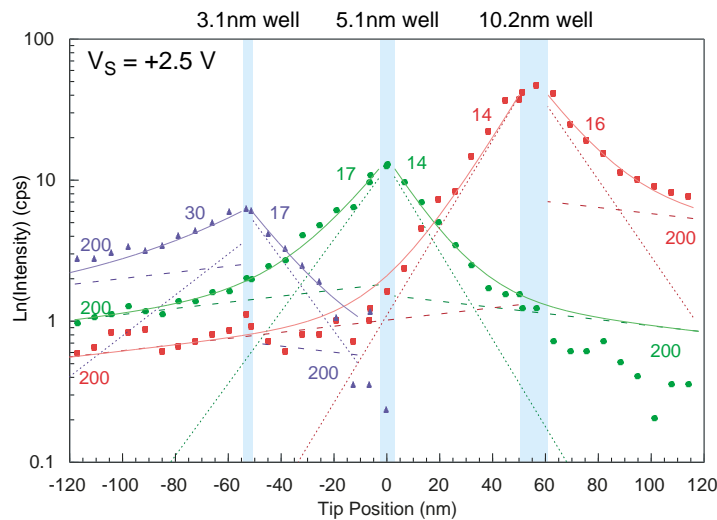

Figure 5. Logarithmic plot of emission intensity from the three wells as a function of tip position for $\mathrm{V}_{S}=+2.5 \mathrm{~V}$. The solid squares, circles, and triangles represent the data for the wells of $10.2,5.1$, and $3.1 \mathrm{~nm}$ widths. The dotted and dashed lines are the exponential decay functions with different decay constants $L_{1}$ and $\mathrm{L}_{2}$. The solid curves are the superpositions of the dotted and dashed lines.

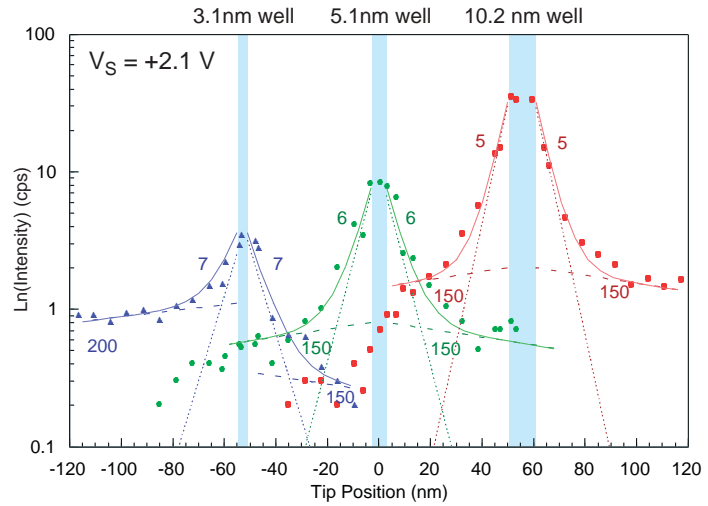

Figure 6. Logarithmic plot of emission intensity from the three wells as a function of tip position for $\mathrm{V}_{S}=+2.1 \mathrm{~V}$. The data are fitted by the theoretical decay curves with two distinct decay constants in the same manner as in Fig. 5.

\section{Conclusion}

In conclusion, we have demonstrated that the thermalization and diffusion lengths of injected electrons in QW structures 
can be measured in real space by using the atomic-scale spatial resolution of the STM light emission spectroscopy. This technique is very powerful in evaluating nanostructures relevant to nanotechnology.

\section{Acknowledgement}

We gratefully acknowledge valuable advice from Prof. J. Nishizawa. We are also indebted to members of our research group, Y. Uehara, K. Sakamoto, and R. Arafune for their cooperation.

\section{References}

[1] T. Tsuruoka, Y. Ohziumi, S. Ushioda, Y. Ohno, and H. Ohno, Appl. Phys. Lett. 73, 1544 (1998).
[2] T. Tsuruoka, Y. Ohizumi, R. Tanimoto, and S. Ushioda, Appl. Phys. Lett. 75, 2289 (1999).

[3] T. Tsuruoka, Y. Ohizumi, and S. Ushioda, Appl. Phys. Lett. 82, 3257 (2003); J. Appl. Phys. 95, 1064 (2004).

[4] Y. Uehara, T. Matsumoto, and S. Ushioda, Solid State Commun. 122, 451 (2002); Phys. Rev. B 66, 075413 (2002).

[5] K. Sakamoto, K. Meguro, R. Arafune, M. Sato, Y. Uehara, and S. Ushioda, Surf. Sci. 502-503, 149 (2002).

[6] T. Tsuruoka, R. Tanimoto, Y. Ohizumi, R. Arafune, and S. Ushioda, Appl. Phys. Lett. 80, 3748 (2002); Appl. Surf. Sci. 190, 275 (2002).

[7] T. Tsuruoka, H. Hashimoto, Y. Ohizumi, and S. Ushioda, Inst. Phys. Conf. Ser. 174, 61 (2003). 